\section{Nurse prescribing in glaucoma}

\begin{abstract}
Aims/purpose To investigate a nurse-led assessment clinic of new referrals of possible glaucoma. To determine the potential for nurse prescribing. To review the background literature about nurse prescribing. Methods Audit of the outcomes of patients attending the nurse-led glaucoma assessment clinic during two defined periods (169 patients). An audit of all patients started on timolol or latanoprost treatment by a nurse following a protocol.

Results A total of 46 patients were commenced on treatment at the clinic, 31 on timolol, 14 on latanoprost, and one on brimonidine. Four of these had the treatment stopped at the review clinic in order to reassess the diagnosis. Four patients in the timolol group developed side effects requiring a change in medication, but these could not have been predicted from their past medical history. Nine patients had treatment changed or added to because the intraocular pressure was felt to be inadequately controlled. During the two 3-month audits, a further 11 patients were commenced on treatment for glaucoma at the review clinic.

Conclusions Initial data from this clinic suggest that nurses possess the diagnostic skills necessary to prescribe for new glaucoma patients. The legal and administrative frame works are developing for more nurses to be able to prescribe. With the newer prostaglandin treatments for glaucoma being available, nurses may usefully and safely be able to prescribe first-line treatments for glaucoma.

Eye (2003) 17, 47-52. doi:10.1038/sj.eye.6700355
\end{abstract}

Keywords: glaucoma; nurse prescribing; latanoprost; timolol

\section{Introduction}

It has been estimated that $15 \%$ of new referrals to the hospital eye service either have, or are suspected to have, glaucoma. ${ }^{1}$
ZK Johnson, PG Griffiths and MK Birch ${ }^{a}$

In order to more effectively deal with new referrals with possible glaucoma, a nurse-led glaucoma assessment clinic (GAC) was started at the Royal Victoria Infirmary, Newcastle upon Tyne in May 2000. The aims of the clinic were to promptly see patients newly referred with possible glaucoma, to work up patients so that appropriate information required to make a diagnosis was available, and to use this information to divide patients into diagnostic categories. This enables patients to be triaged so that the most urgent have earlier clinic appointments. It quickly became apparent that significant numbers of patients had advanced high-pressure glaucoma, and the possibility of nurses commencing treatment at the first visit was raised.

We report the results of a review of the first 12 months of this clinic. The first aim was to validate our protocol for nurse prescribing of topical glaucoma medication for newly diagnosed patients with elevated intraocular pressure (IOP). We also assessed the accuracy of the nurses' diagnoses.

\section{Materials and methods}

All patients of participating consultants referred with suspected glaucoma were selected for the GAC unless the letter suggested that they might have media opacities or other reasons for the diagnosis to be difficult.

Three experienced ophthalmic staff nurses, (F and E grades) all currently working in nurse practitioner roles within eye outpatients, were recruited to work in the GAC. As such they were already competent using the slit lamp, applanation tonometry, and fundal examination. Specific training in optic disc assessment was given. This included seeing patients in parallel with a consultant until they were felt to be of an appropriate standard.

Validation was achieved by audit of diagnostic accuracy after 3 months.

The nursing staff completed a proforma (adapted from one developed for use at Sunderland Eye Infirmary with kind permission from Mr P Phelan), including information on ophthalmic, general medical, and family history,
${ }^{a}$ Department of Ophthalmology Royal Victoria Infirmary Newcastle upon Tyne, UK

Correspondence:

MK Birch

Department of

Ophthalmology

Claremont Wing

Royal Victoria Infirmary

Queen Victoria Road

Newcastle upon Tyne NE1 4LP, UK

Tel: + 4401912825448

Fax: + 4401912275246

E-mail: birchmk@ aol.com

Received: 13 November 2001

Accepted in revised form: 2 July 2002

This work was done as part of an MPhil degree, part funded by an educational grant from Pharmacia \& Upjohn Ltd 
and current medication, for all patients attending the GAC. Examination consisted of Goldmann applanation tonometry, threshold perimetry using the

24-2 programme with Swedish Interactive Thresholding Algorithm (SITA) on a Humphrey visual field analyser (Zeiss Humphrey Systems, Dublin, CA, USA), and assessment of the optic disc after mydriasis using a $90 \mathrm{D}$ lens and disc photographs. The proforma required assessment of cup : disc ratio and focal disc pathology as part of a clinical decision by the nurse as to whether a disc was glaucomatous or not. The level of the IOP, presence of a visual field defect as determined by the glaucoma hemifield test (GHT), and the optic disc appearance were used to allocate patients to one of five

Table 1 Diagnostic categories used in the GAC

\begin{tabular}{ll}
\hline Glaucoma & $\begin{array}{l}\text { IOP 22 mmHg or over, field defect, and } \\
\text { cupped disc }\end{array}$ \\
Glaucoma suspect & $\begin{array}{l}\text { Any IOP, equivocal visual field defect, or } \\
\text { disc cupping especially if family history }\end{array}$ \\
Normal tension & $\begin{array}{l}\text { IOP 21 mmHg or less, visual field defect, } \\
\text { and cupped disc } \\
\text { glaucoma }\end{array}$ \\
$\begin{array}{l}\text { Ocular } \\
\text { hypertension }\end{array}$ & $\begin{array}{l}\text { IOP 22 mmHg or more, normal visual field, } \\
\text { and normal disc }\end{array}$ \\
& $\begin{array}{l}\text { IOP 21 mmHg or less, normal field, and } \\
\text { normal disc }\end{array}$ \\
\hline
\end{tabular}

diagnostic categories: normal, glaucoma, glaucoma suspect, normal tension glaucoma, and ocular hypertension, using the definitions in Table 1. By following a flow pathway, the treatment and follow-up arrangements were then decided (Chart 1).

The nurse in the GAC prescribed according to a written protocol using timolol 0.5\% ('Timoptol', Merck \& Co., Inc., NJ, USA) or latanoprost ('Xalatan', Pharmacia Corporation, NJ, USA) where beta-blockers were contraindicated, for example by a history of a heart or chest condition. The protocol was agreed between medical and nursing staff and, as this was a pilot scheme, a member of the medical staff checked the nurses decision against the protocol before prescribing. Patients with IOPs of $22-40 \mathrm{mmHg}$, disc cupping, and field loss (glaucoma category as in Table 1) were prescribed ocular hyoptensives. No patient had more than one medication prescribed at this visit. If treatment was started, the patients had a review clinic appointment within 1 month.

We audited the GAC using the medical review clinic as the gold standard. This comprised two periods when all patients attending the GAC were audited (May-August 2000, and February-April 2001). Additionally, all patients diagnosed with glaucoma and consequently started on treatment by the nurses over the full 12-month period (May 2000-May 2001) were also audited.

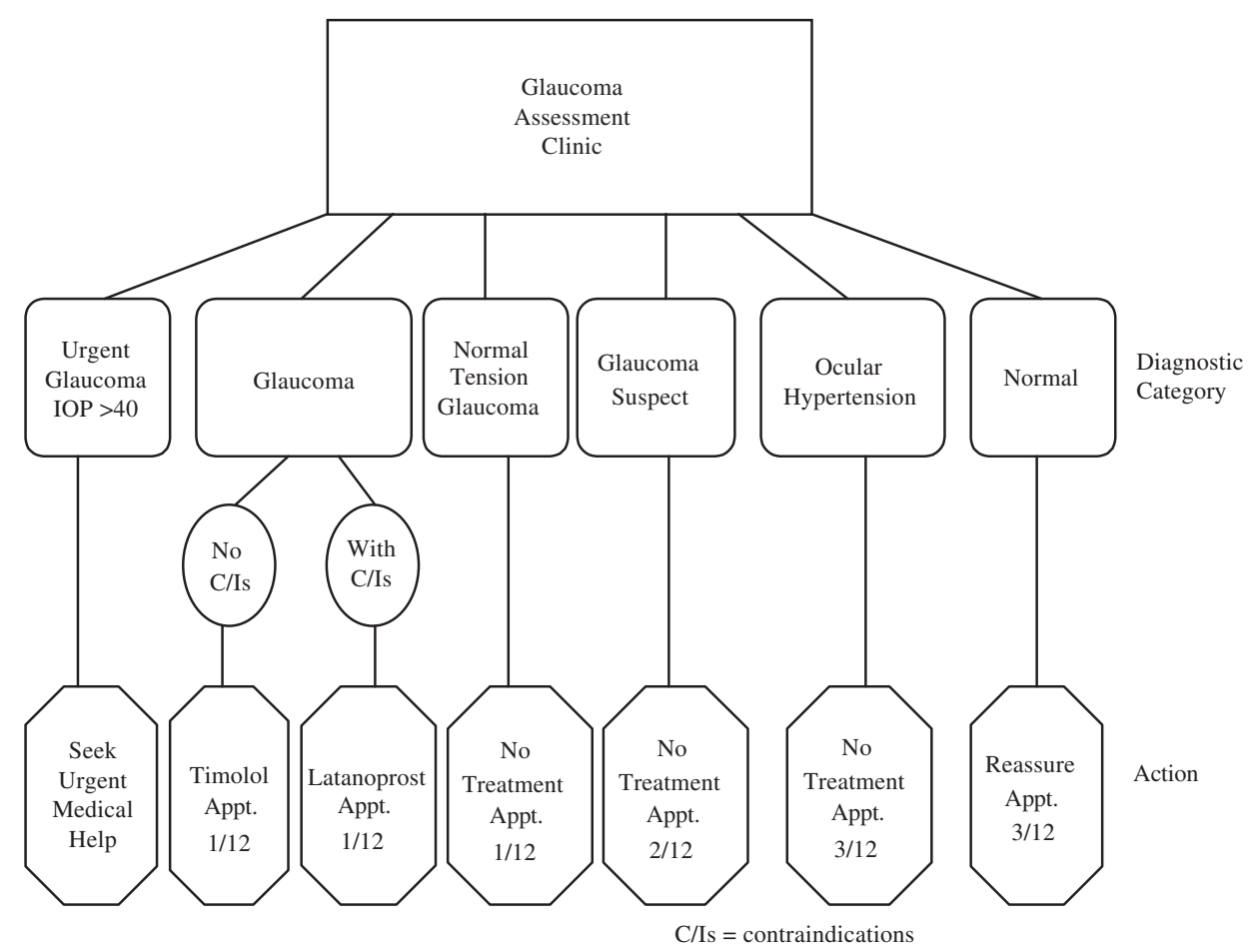

Chart 1 Flow pathway for treatment and follow-up arrangements. 


\section{Results}

\section{Diagnostic accuracy}

Over the first 12 months of the glaucoma assessment clinic, 378 patients were seen and 52 patients were put into the 'glaucoma' category (13.8\%). Of these, two had narrow angles requiring YAG peripheral iridotomies, two were already known to have glaucoma (diagnosed elsewhere), and two were brought to the next consultant clinic for confirmation of the diagnosis. This left 46 $(12.2 \%)$ patients in this category who were commenced on treatment by the nurses at the GAC. Four of these patients had their treatment stopped at the medical review clinic, giving a false-positive rate of $8.7 \%(95 \%$ confidence interval $0.55-16.85 \%$ ): one patient with spuriously high pressure owing to squeezing and artefactual visual field loss, and three patients owing to artefactual visual field loss.

The two audits consisted of 169 patients. In addition to those identified at the GAC, a further 11 patients $(6.5 \%)$ were diagnosed as glaucoma at the subsequent review clinic and required treatment $(6.5 \%$ false-negative rate, 95\% confidence interval $2.8-10.2 \%$ ). If this proportion was consistent over the year, that would be a further 25 patients $(11 / 169 \times 378)$ with open-angle glaucoma requiring treatment, or a total of 71 patients out of the 378 original referrals $(18.8 \%)$. The numbers in each of these diagnostic categories during the audit periods are shown in Table 2, together with the subsequent diagnosis determined at the medical review clinic. The median IOP for each group is also shown. The age range of the treated patients was $47-92$ years. The median IOP of the eyes treated was $32 \mathrm{mmHg}$; the spread of IOPs is shown in Chart 2.

The one patient originally in the normal category who was subsequently diagnosed with glaucoma would not have fitted the protocol definition of glaucoma even on the second visit; the doctor concerned did not use the protocol definition at the review clinic.

The nurses could not categorise four patients according to the protocol. These patients were brought to the next consultant's clinic, and the ultimate diagnoses are shown in Table 2.

\section{Treatment protocol}

A total of 31 patients had been commenced on timolol, 14 on latanoprost, and one on brimonidine ('Alphagan', Allergan, Irvine, CA, USA). The casualty doctor over-ruled the protocol on this occasion. Of the 31 started on timolol, five had latanoprost added at the review clinic visit because control was not good enough, and eight were switched to latanoprost as monotherapy. Of these eight, four were switched because of poor control and four owing to side effects. None of the patients started on latanoprost had their treatment altered.

Table 2 GAC findings and diagnosis compared with subsequent medical review (both 3-month audit periods combined $n=169$ )

\begin{tabular}{|c|c|c|}
\hline GAC category/median IOP & Number & Diagnosis in medical review clinic \\
\hline Glaucoma suspect/19 mmHg & 79 & $\begin{array}{l}9 \text { glaucoma } \\
35 \text { normal ( } 8 \text { physiological cupping; } 4 \text { artefactual defect; } 1 \text { tilted disc) } \\
14 \text { ocular hypertension } \\
13 \text { glaucoma suspect } \\
5 \text { normal tension glaucoma } \\
1 \text { BRVO; } 2 \text { did not attend follow-up }\end{array}$ \\
\hline Glaucoma/32 mmHg & 28 & $\begin{array}{l}24 \text { glaucoma } \\
2 \text { glaucoma suspect } \\
1 \text { ocular hypertension } \\
1 \text { normal }\end{array}$ \\
\hline $\begin{array}{l}\text { Normal tension glaucoma/ } \\
17 \mathrm{mmHg}\end{array}$ & 24 & $\begin{array}{l}10 \text { normal tension glaucoma } \\
6 \text { glaucoma suspect } \\
5 \text { normal ( } 2 \text { physiological cupping; } 1 \text { artefactual; } 1 \text { tilted) } \\
1 \text { cataract; } 1 \text { chorioretinal scar; } 1 \text { did not attend }\end{array}$ \\
\hline $\begin{array}{l}\text { Ocular hypertension/ } \\
27 \mathrm{mmHg}\end{array}$ & 15 & $\begin{array}{l}11 \text { ocular hypertension } \\
1 \text { glaucoma } \\
3 \text { did not attend }\end{array}$ \\
\hline Normal/16 mmHg & 19 & $\begin{array}{l}7 \text { normal } \\
4 \text { ocular hypertension } \\
3 \text { glaucoma suspect } \\
1 \text { primary open-angle glaucoma } \\
4 \text { did not attend }\end{array}$ \\
\hline Noncategorised patients & 4 & 1 neurological field loss; 1 tilted disc; 1 disc drusen+OHT; 1 OHT+macular hole \\
\hline
\end{tabular}

\footnotetext{
${ }^{a}$ Patient not diagnosed using the GAC definition.
} 


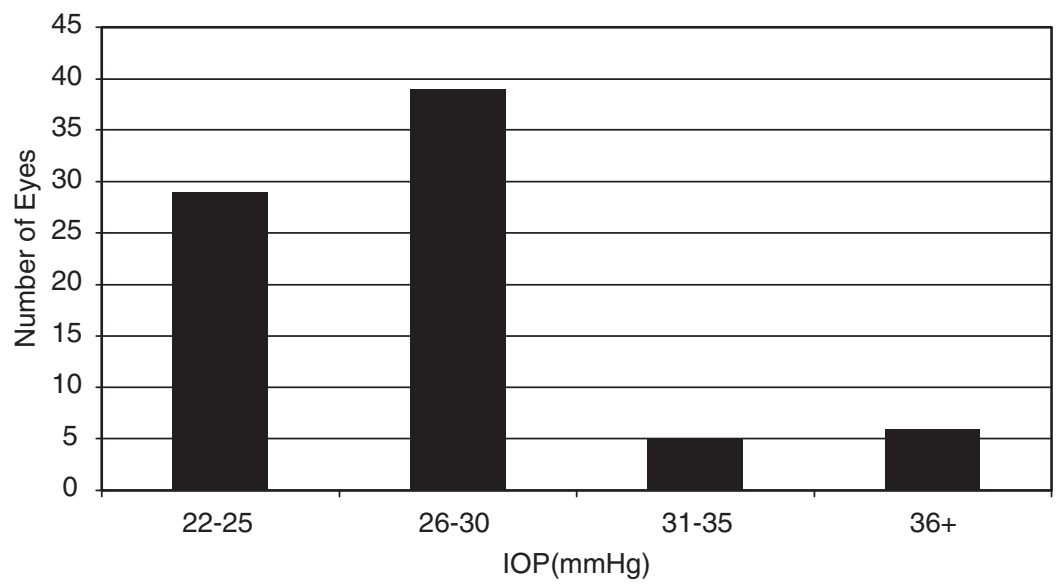

Chart 2 Range of intraocular pressure in the treated group.

The patient started on brimonidine had latanoprost added owing to poor control.

As already mentioned, in four patients the treatment was stopped in order to reassess the diagnosis. Two were recategorised as 'glaucoma suspect': in one the field defect was thought to be a result of cataract and in the other the disc cupping was thought to be 'physiological' and the visual field loss equivocal. In this patient the positive GHT was felt to be artefactual. One case was recategorised as 'ocular hypertension with a tilted disc'. In the preceding three cases, the IOP was again elevated after treatment was discontinued. The fourth patient initially had IOPs of 26 and $40 \mathrm{mmHg}$, but on subsequent review the pressures were normal off treatment and the field normal; the initial IOPs appear to have been spuriously high, possibly owing to attempted forcible lid closure.

No topical side effects of the treatments were noted. The systemic side effects encountered were all in the timolol-treated patients. Two complained of low energy and one of wheezing. One patient developed dyspnoea, which required a visit to the eye casualty before the review appointment. None had contraindications identifiable from their past medical history, and the symptoms resolved on changing the drops.

\section{Discussion}

\section{Nurse prescribing}

The developing framework for the facilitation of nurse prescribing within the NHS has centred on the reports of two advisory groups chaired by Dr June Crown. ${ }^{2-4}$ The first report in $1990^{2}$ recommended that some district nurses and health visitors should be able to prescribe from a limited formulary. The resulting Medicinal Products: Prescription by Nurses Act came into effect in $1994 .^{5}$
The second more extensive review looked at the whole process of prescribing across the NHS. ${ }^{4}$ As a result, an interim report on group protocols was published in April 1998. ${ }^{3}$ Group protocols had become the main mechanism for nurse prescription in hospitals, but there was no agreed national standard.

At present, only district nurses and health visitors have been able to undergo training to become nurse prescribers. Last year, proposals were announced to extend the scheme to include other medical situations and groups of nurses. ${ }^{6}$ Training programmes for the extended prescribing scheme are due to start in $2002{ }^{7}$ Nurses completing this course will be able to prescribe all General Sales List and Pharmacy Medicines currently prescribable by GPs and also a list of Prescription Only medicines. This will allow these nurses to manage a range of specific medical conditions in the areas of minor ailments and injuries, chronic illness, health promotion, and palliative care. ${ }^{7}$ Two of the main factors suggested for deciding who should be trained to prescribe are the medical condition and an NHS service need, with nurse-led clinics being an area where an early benefit to patients may be seen. ${ }^{6}$

In a press release in May 2001, the Government announced its intention to allow 'supplementary' prescribing. This would enable nurses to treat more complex medical conditions such as asthma, diabetes mellitus, hypertension, and coronary heart disease, as well as mental health. ${ }^{8}$ Supplementary prescribing is now a key element of government NHS reforms with a target of 30000 nurse prescribers by $2004 .^{9}$

Currently, true nurse prescribing involving the 'authorising in writing of the supply of a named medicine for a named patient ${ }^{3}$ can only occur in the community setting. In this situation, a nurse can write a prescription that is accepted by a pharmacist, who then dispenses the item. There has been a report of a local 
arrangement for a hospital pharmacy to accept a nurse's signature on a special prescription form for a limited list of items in a dermatology department, ${ }^{10}$ but this formal arrangement is unusual. In the hospital setting, nurse prescribing tends to overlap with the 'supply' (provide a medicine for a patient or carer to administer) and 'administering' (to give a medicine to a patient) of a medicine. $^{3}$

Currently, nurses can prescribe in the hospital setting by the following means:

1. Medical countersignature: This is probably the most common occurrence where nurses effectively make the prescribing decision but a doctor actually writes the prescription. This has long been common in the provision of inpatient pain relief. Likewise, nurses working in eye casualties often prescribe chloramphenicol ointment for corneal abrasions in this way.

2. Standing order: This is drawn up by a department for nurses to administer a single dose of a particular medication to all patients in a specific situation. It is signed by all consultants in the department and renewed annually. Standing orders are commonly used to allow nurses to administer preoperative topical mydriatics.

3. Group protocol: This is a mechanism to allow nurses to initiate or change treatment for a specific condition according to criteria agreed in advance by the department involved. Increasingly, group protocols are being drawn up to allow nurses to supply postoperative drops after cataract surgery.

Dermatology is one of the few hospital specialities to have published on their use of nurse prescribing. A survey of dermatology departments published in 1999 revealed the wide use of nurse-run clinics and the use of protocols for nurses to alter treatments (57\%) or initiate them $(26 \%) .{ }^{11}$ Cox and Walton ${ }^{10}$ reported that nurses make treatment choices closer to those of the consultants than their nonspecialist junior medical staff.

\section{Potential role for nurse prescribing in glaucoma}

Glaucoma has a number of features that make it suitable for nurse prescribing. It is a common chronic condition with a large service impact. There are also established clinics run by nurses or paramedical staff. The final requirement for nurse prescribing is an effective and safe drug. Evidence now suggests that the prostaglandin eye drop latanoprost may actually be a more effective ocular hypotensive than timoptol, ${ }^{12}$ while offering a superior systemic safety profile. ${ }^{13}$ It has therefore had its license changed to allow first-line use, and consequently appears to be a suitable treatment for nurses (and/or other paramedical staff) involved in glaucoma care to initiate treatment with. There is current debate as to the safety of allowing nurses to prescribe recently licensed black triangle drugs. ${ }^{9}$ This would apply to the newly licensed ocular hypotensive lipid bimatoprost ('Lumigan', Allergan) and prostaglandin analogue travoprost ('Travatan', Alcon, Fort Worth, TX, USA), which may have similar efficacy and side-effect profiles to latanoprost.

Although numbers are small, our study showed that patients who were prescribed latanoprost had more satisfactory control at the first review visit and none of them had suffered any side effects. This is in contrast to those who received timolol initially, with nine patients having inadequate control and four suffering side effects in the first month.

Nurse-run clinics can be, and are being, used in two areas of the care of patients with glaucoma. One is the initial assessment of patients with possible glaucoma and the other is the regular review of 'stable' patients. ${ }^{14}$

Referrals of patients with possible glaucoma include a wide range, from those with advanced disease, through ocular hypertensives, to a large number who are found to be normal but had an artefactually high IOP or a suboptimal visual field test at the optometrists. If the patients with advanced disease can be recognised, and those who are probably normal reassured, it is possible to prioritise the timing of the follow-up appointment at the consultant's clinic.

If a newly diagnosed patient requires treatment, it is more efficient to do this at the assessment clinic. This means that the IOP is lowered as quickly as possible and the effectiveness of the treatment can be reviewed when the patient attends the follow-up consultants clinic, allowing the earlier switch or addition of treatment. While raised IOP is not central to the diagnosis of glaucoma, it is the only treatable risk factor. Evidence for the effectiveness of reducing IOP in halting disease progression is still not complete, but as higher IOPs are associated with faster progression of visual field defects, ${ }^{15}$ it seems logical that a patient presenting with high-pressure glaucoma should have their pressure controlled as soon as possible.

The role of nurse prescribing is clearly not to initiate early treatment for borderline cases of glaucoma or ocular hypertensives. Starting treatment at assessment removes the possibility of a recheck of untreated IOP at the consultants clinic, which may be useful for the decision-making process in the management of such patients. For this reason, patients diagnosed as normal tension glaucoma were also not considered for nurse treatment but given early medical review (Chart 1 ). However, our experience is that about $12 \%$ of referrals have advanced high-pressure glaucoma and must be considered to benefit from the earliest possible treatment 
when presenting at such a clinic. Chosen nurse treatment IOP threshold of $22 \mathrm{mmHg}$ could of course be varied.

In total, about two-thirds of the patients identified at the consultants review clinic as requiring treatment had been commenced on it at the nurse assessment clinic.

More importantly, no patients requiring urgent treatment were missed (ie those with high pressure and moderateto-severe visual field loss). Borderline IOPs and/or disc assessment were the main reasons for patients, with generally milder glaucoma, not being identified and treated by the nurses at the GAC. One patient was started on treatment for what subsequently turned out to be spuriously high IOP readings, possibly owing to squeezing, and artefactual visual field loss. With hindsight, the high pressure appeared to have caused the nurse to overlook the borderline appearance of the optic discs. This underlines the need for the protocol to be tight and strictly adhered to, and for the nurses to have a clear understanding of their role in starting treatment. The three other patients in whom treatment was stopped at the second visit appeared to fit the criteria for treatment according to the examination record of the first visit.

It is apparent that the criteria for identifying visual field loss need to be improved. The single field test at the GAC meant that 'learning effect' was a potential confounding factor. In practice, this did not appear to be significant in the diagnosis of moderate-to-severe disease most appropriate to nurse prescribing. Over-reliance on objective statistical determination of glaucomatous loss by GHT was inadequate. We feel a broader understanding of clinical visual field interpretation is where further training should most usefully be directed. Ongoing developments in optic disc imaging may also be a future means of improving accuracy in nurse diagnosis.

There is a cost associated with a nurse assessment clinic. However, in our experience, in addition to potential improvements in quality of care, this cost may also be partially offset by resulting efficiencies in follow-up. A significant number of normals were initially classified as glaucoma suspect at the GAC and it is important that such patients are informed in such a manner as to avoid causing unnecessary anxiety while they await medical review.

The current climate is favourable for the expansion of nurse prescribing. Our results show that nurses can identify the significant number of patients with moderate-to-severe high-pressure disease still being referred from the community and requiring urgent treatment. Particularly if latanoprost is used, nurse prescribing in glaucoma can be a safe and effective option. The addition of glaucoma to the list of conditions, where prescribing by trained ophthalmic nurses can occur, seems appropriate, and further investigation is required.

\section{Acknowledgement}

We thank Mr RC Bosanquet and Sister G Charlton.

\section{References}

1 Harrison RJ, Wild JM, Hobley AJ. Referral patterns to an ophthalmic outpatient clinic by general practitioners and ophthalmic opticians and the role of these professionals in screening for ocular disease. BMJ 1988; 297: 1162-1167.

2 Crown J. Report of the advisory group on nurse prescribing. Department of Health: London, 1990.

3 Crown J. Report on the supply and administration of medicines under group protocols. Department of Health: London, 1998.

4 Crown J. Review of prescribing, supply and administration of medicines. NHS Executive (Department of Health): London, 1999.

5 Green J. Development of the nurse prescribing initiative. In: Humphries JL, Green J (eds). Nurse Prescribing, Macmillan Press Ltd: New York, 1999.

6 Robinson P. Consultation on proposals to extend nurse prescribing. Department of Health: London, 25th October 2000.

7 Department of Health. Nurse prescribing, 2001, webpage: www.doh.gov.uk/nurseprescribing.

8 Department of Health. Press Release ref. 2001/0223, 2001, www.doh.gov.uk

9 Horton R. Nurse-prescribing in the UK: right but also wrong. Lancet 2002; 359: 1875-1876.

10 Cox NH, Walton Y. Prescribing for out-patients by nursing staff in a dermatology department. Br J Dermatol 1998; 139: 77-80.

11 Cox NH. The expanding role of nurses in surgery and prescribing in British departments of dermatology. Br J Dermatol 1999; 140: 681-684.

12 Zhang WY, Po ALW, Dua HS, Azuara-Blanco A. Meta-analysis of randomised controlled trials comparing latanoprost with timolol in the treatment of patients with open angle glaucoma or ocular hypertension. $\mathrm{Br} J$ Ophthalmol 2001; 85: 983-990.

13 Watson PG. Latanoprost: two years experience of its use in the United Kingdom. Latanoprost Study Group. Ophthalmology 1998; 105: 82-87.

14 Hume J, Abbott F. Setting up a shared care glaucoma clinic. Nurs Stand 1995; 10: 34-36.

15 Jay JL, Murdoch JR. The rate of visual field loss in untreated primary open angle glaucoma. Br J Ophthalmol 1993; 77: 176-178. 